\title{
Editor's Note/ Acknowledgements
}

For this issue of Constellations, we implemented changes in our review process, which posed a few challenges for the publication. We would like to thank the Department of History and Classics (Jessica, Melanie, \& Sheryl) and our Faculty of Advisor, Professor Jeremy Caradonna. Most importantly, we would like to thank the authors and the reviewers who put in their valuable time and patiently worked with the Editorial Board in face of the changes we implemented.

\section{Editors}

Ezekiel Gow

Dongwoo Kim

\section{Assistant Editors}

Erin Gallagher-Cohoon

Kayla Reddecliff

\section{Senior Reviewers}

Merissa Daborn

Charlotte Forss

Kaitlyn Grant

Jordan Hupka

Edward Middleton

Karey Rodgirs

\section{Faculty Advisor}

Jeremy Caradonna 